OPEN ACCESS

Edited by:

Qingfeng Zhu,

Johns Hopkins Medicine,

United States

Reviewed by:

Zsolt Kovács,

George Emil Palade University of Medicine, Pharmacy, Sciences and

Technology of Târgu Mureş, Romania Ding Ding,

The Johns Hopkins Hospital, United States

*Correspondence: Guanglin $\mathrm{Cu}$ guanglin.cui@nord.no

Specialty section:

This article was submitted to Gastrointestinal Cancers, a section of the journal Frontiers in Oncology

Received: 30 June 2020 Accepted: 25 January 2021 Published: 05 March 2021

Citation:

Cui G (2021) The Mechanisms Leading to Distinct Responses to PD-

1/PD-L1 Blockades in Colorectal Cancers With Different MSI Statuses,

Front. Oncol. 11:573547. doi: 10.3389/fonc.2021.573547

\section{The Mechanisms Leading to Distinct Responses to PD-1/PD-L1 Blockades in Colorectal Cancers With Different MSI Statuses}

\author{
Guanglin Cui ${ }^{1,2 *}$ \\ ${ }^{1}$ Research Group of Gastrointestinal Diseases, The Second Affiliated Hospital of Zhengzhou University, Zhengzhou, China, \\ 2 Faculty of Health Science, Nord University, Bodø, Norway
}

Current clinical studies showed distinct therapeutic outcomes, in which CRC patients with mismatch repair-deficient (dMMR)/microsatellite instability high (MSI-H) seem to be relatively more "sensitive" in response to anti-programmed death-1 receptor (PD-1)/ programmed death-1 receptor ligand 1 (PD-L1) therapy than those with mismatch repairproficient (pMMR)/microsatellite instability-low (MSI-L). The mechanisms by which the same PD-1/PD-L1 blockades lead to two distinct therapeutic responses in CRC patients with different MSI statuses remain poorly understood and become a topic of great interest in both basic research and clinical practice. In this review of the potential mechanisms for the distinct response to PD-1/PD-L1 blockades between dMMR/MSI-H CRCs and PMMR/MSI-L CRCs, relevant references were electronically searched and collected from databases PubMed, MEDLINE, and Google scholar. Sixty-eight articles with full text and 10 articles by reference-cross search were included for final analysis after eligibility selection according to the guidelines of PRISMA. Analysis revealed that multiple factors e.g. tumor mutation burden, immune cell densities and types in the tumor microenvironment, expression levels of PD-1/PD-L1 and cytokines are potential determinants of such distinct response to PD-1/PD-L1 blockades in CRC patients with different MSI statuses which might help clinicians to select candidates for anti-PD-1/PDL1 therapy and improve therapeutic response in patients with CRC.

Keywords: therapy, inhibitor, immune checkpoint, metastasis, colorectal cancer

\section{INTRODUCTION}

Colorectal cancer (CRC) is the third most common type of cancers and the second leading cause of cancer-related death in developed countries. Although numerous attempts have been made to increase the overall survival rate of CRC patients, the improved prognosis in CRCs is still heavily dependent on early diagnosis and complete resection of the primary tumor. Unfortunately, 
metastasis is often observed at the time of diagnosis, and curative surgical resection becomes impossible in these patients. Thus, to improve the prognosis of metastatic CRC (mCRC) patients, many researchers have investigated the escape immunosurveillance mechanisms by which the CRC progresses and metastases persistently (1-4). A growing body of evidence from various studies has demonstrated that immune checkpoints are negative regulators for host anti-tumor immune function and could significantly suppress the host anti-tumor immune reactivity (5-7). Therefore, one of the proposed mechanisms for a tumor's ability to escape immunosurveillance is by targeting of immune checkpoint molecules and becomes an important therapeutic strategy for the treatment of human cancers. Indeed, recent studies revealed that immune checkpoint blockades with monoclonal antibodies (mAbs) that target the programmed cell death receptor 1 (PD-1) and its ligand (PD-L1) have been widely used in treating human cancers and showing in an enhanced host anti-tumor immunity and increase survival rates in a variety of malignancies including melanoma (8), renal cell carcinoma (9), and non-small cell lung cancer (10). The therapeutic efficacy of anti-PD-1/PD-L1 mAbs has been evaluated in patients with solid tumors including mCRC (1114). However, unlike the efficacy reported in patients with melanoma, the therapeutic response of anti-PD-1/PD-L1 mAbs in patients with mCRC is very different between mismatch repair-deficient (dMMR)/microsatellite-instability-high (MSI-H) tumors and mismatch repair-proficient (pMMR)/microsatelliteinstability-low (MSI-L) or microsatellite-stability (MSS) tumors $(11,15-18)$. Results of clinical studies or trials showed that CRC patients with $\mathrm{dMMR} / \mathrm{MSI}-\mathrm{H}$ seem to be relatively more "sensitive" in response to anti-PD-1/PD-L1 mAbs than those CRCs with pMMR/MSI-L: dMMR/MSI-H CRCs treated with anti-PD-1/PD-L1 mAbs exhibit significantly enhanced sustained clinical responses. However, anti-PD-1/PD-L1 therapies are ineffective in CRC patients with pMMR/MSI-L $(12,13,19)$. The mechanisms leading to distinct therapeutic outcomes between dMMR/MSI-H CRCs and pMMR/MSI-L CRCs have been a topic of great clinical interest and studied.

Therefore, this manuscript reviews the current understanding of the potential mechanisms leading to a distinct therapeutic response to anti-PD-1/PD-L1 mAbs between dMMR/MSI-H CRCs and pMMR/MSI-L CRCs.

\footnotetext{
Abbreviations: APC, antigen-presenting cells; PRISMA, Preferred reporting items for systematic reviews and meta-analyses; CAMK1D, the calcium/calmodulindependent protein kinase 1D; CRC, colorectal cancer; CTL, cytotoxic T lymphocyte; CTLA4, cytotoxic T-lymphocyte associated protein 4; dMMR, mismatch repair-deficient; IDO, indolamine $2^{\prime} 3^{\prime}$-dioxygenase; IFN- $\gamma$, interferon- $\gamma$, IL. Interleukin; $\mathrm{mAb}$, monoclonal antibody; mCRC, metastatic CRC; MDSCs, myeloid-derived suppressor cells; MHC, major histocompatibility complex; MSI-H, microsatellite-instability-high; MSI-L, microsatellite-instabilitylow; NK, natural killer; ORR, the objective response rate; OS, overall survival; PD1 , programmed death-1 receptor; $\mathrm{PD}-\mathrm{L} 1$, programmed death-1 receptor ligand; PFS, progression-free survival; pMMR, mismatch repair-proficient; TGF- $\beta$, transforming growth factor- $\beta$; TIL, tumor infiltrating lymphocyte; TIME, tumor immune microenvironment; TMB, tumor mutational burden; TNF- $\alpha$, tumor necrosis factor- $\alpha$; Treg, regulatory $\mathrm{T}$ cell.
}

\section{SEARCH STRATEGY AND SELECTION CRITERIA}

\section{Literature Search Strategy}

Relevant electronic literature search was conducted in academic databases PubMed, MEDLINE and Google scholar by the author using the search terms "anti-PD-1", "anti-PD-L1", "immune checkpoint inhibitor", "response", "predicators", "colorectal cancer", "metastasis", "dMMR", "pMMR" and "MSI" from inception to September 2020. Of 270 abstracts hit, sixty-eight articles with full text were included for final analysis according to the guidelines of Preferred Reporting Items for Systematic Reviews and Meta-Analyses (PRISMA) (see Figure 1). Ten articles selected from reference lists of appropriate papers as an additional literature source were included.

\section{Inclusion and Exclusion Criteria}

The following selection criteria were used: (1) articles published in English; (2) full-text available; (3) studies or trials conducted in human CRCs; (4) relevant mechanistic studies conducted in mice; (5) articles only published as abstract or non-full text publications (case reports, editorials, letters to editors, or meeting abstracts) were excluded; (six) studies were rejected if they lacked sufficient information to study anti-PD-1/PD-L1 therapies in CRC.

The following information was extracted: year of publication; country of origin; study design; journal; study population size; and mean age of patients. A meta-analysis was not carried out because of the heterogeneity, differences in metrics and settings in the included publications.

\section{A BRIEF OVERVIEW OF THE ROLE OF PD-1/PD-L1 IN THE ESCAPE IMMUNOSURVEILLANCE OF CRC}

PD-1 is an inhibitory coreceptor that is highly expressed on the cell surface of various types of immune cells such as T and B lymphocytes and natural killer (NK) cells $(20,21)$. In the tumor microenvironment, PD-1 is also expressed in tumor-infiltrating lymphocytes (TILs) and participates in the modulation of host anti-tumor immune response (20). The modulation effect of PD1/PD-L1 on immune reactivity has been well documented by intensive studies. Physiology, the binding of PD-1 with its ligand PD-L1 leads to an inhibitory efficiency of T cell activation and suppression of cytokine productions, i.e., interferon- $\gamma($ IFN- $\gamma$ ), tumor necrosis factor- $\alpha$ (TNF- $\alpha$ ) and interleukin (IL)-2 from immune cells (22). These effects hamper the overreaction of immune response and help to maintain immune homeostasis. In the tumor microenvironment, PD-L1 is expressed on the tumor cell surface and the binding of PD-1 expressed by TILs may lead to the functional inactivation of TILs and loss of their ability to kill tumor cells that result in immune resistance in patients with tumors (23, 24). Therefore, the PD-1/PD-L1 pathway has been recognized as a negative modulator of immune response by restricting the function of TILs in the tumor immune microenvironment (TIME), and inhibition of the PD-1/PD-L1 pathway by administration of mAbs 


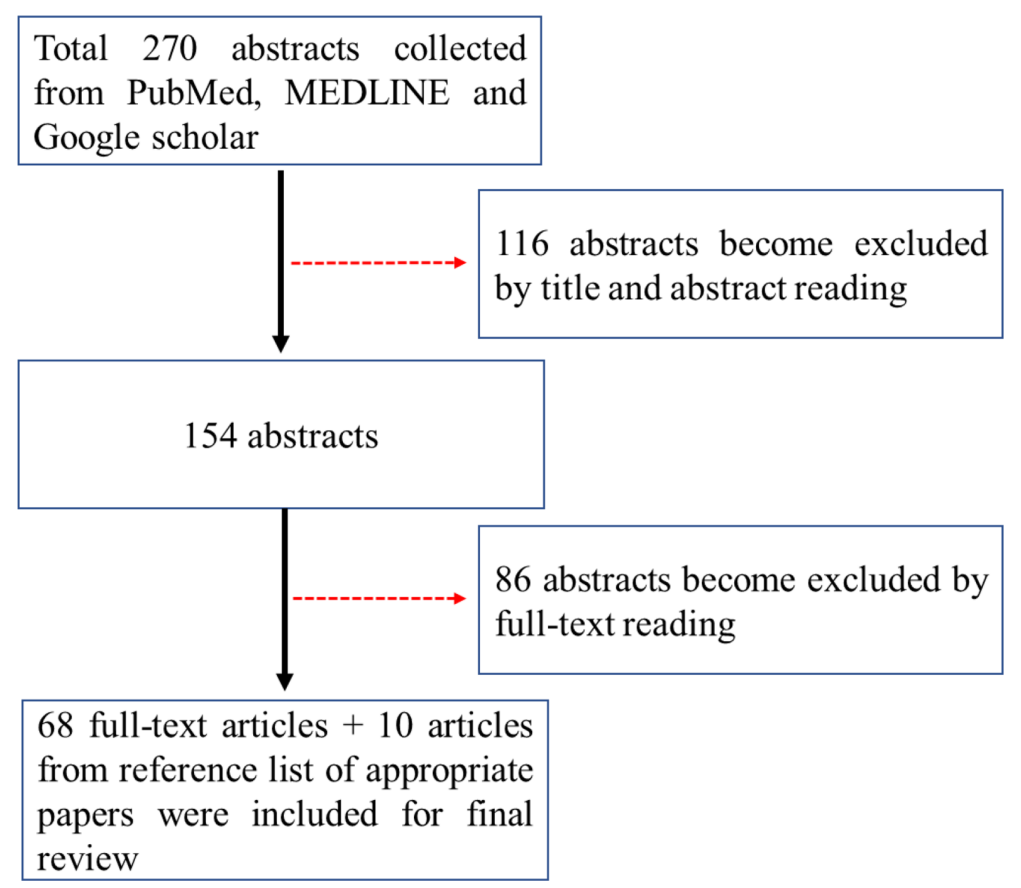

FIGURE 1 | Prisma flow chart for the search of the literatures.

can reactivate the function of cytotoxic T lymphocytes (CTLs) and their ability to attack tumor cells (25).

In addition to anti-PD-1/PD-L1 mAbs, blockades of other immune checkpoint factors such as cytotoxic T-lymphocyte associated protein 4 (CTLA4) mAbs have been shown to induce significantly enhanced $\mathrm{T}$ cell proliferation and IL-2 production $(26,27)$ and decreased density of regulatory $\mathrm{T}$ cells (Tregs) in the TIME $(27,28)$, which relates to an improved prognosis in patients with tumors (7). Taken together, current available scientific evidence suggests that the immune checkpoint PD-1/PD-L1 pathway plays a critical role in the process of immunoediting and tumor progression and metastasis (29). Pharmacologically, PD-1/PD-L1 pathway signals can be easily targeted. Recent immunotherapeutic strategies based on $\mathrm{mAb}$ targeting of immune checkpoint molecules such as PD-1 and its ligand PD-L1 and CTLA4 have shown a promising therapeutic response that leads to increase the functional activation of immune cells and enhanced host anti-tumor immune response in patients with tumors such as malignant melanoma $(8,25)$, renal cell cancer (30), lung cancer (30) and CRC (7).

\section{DISTINCT THERAPEUTIC EFFICACY OF ANTI-PD-1/PD-L1 mAbs BETWEEN PATIENTS WITH dMMR/MSI-H CRC AND PATIENTS WITH PMMR/MSI-L OR MSS CRC}

According to its etiology, CRCs can be divided into sporadic, familial, or hereditary (30). Approximately $70-75 \%$ of CRCs are sporadic CRCs and become the main type of CRCs in the clinic. Clinical studies demonstrated that dMMR/MSI-H status is observed in approximately $15-20 \%$ of sporadic CRCs $(31,32)$; higher immune cell infiltration and better prognosis are more frequently reported in dMMR/MSI-H tumors than pMMR/MSI-L tumors (33). The therapeutic efficacy of anti-PD-1 and anti-PDL1 mAbs in patients with CRCs has been recently evaluated. A distinct therapeutic efficacy of anti-PD-1/PD-L1 mAbs between dMMR/MSI-H tumors and pMMR/MSI-L tumors was demonstrated in numerous clinical studies (15, 16, 30, 34-41). Overman et al. (36) investigated the efficacy and safety of nivolumab (an anti-PD1 $\mathrm{mAb}$ ) combined with ipilimumab (anti-CTLA-4 mAb, another immune checkpoint inhibitor) in patients with MSI-H mCRC vs. non-MSI-H mCRC. They reported that nivolumab as a single bioagent or in combination with ipilimumab could result in $31 \%$ disease control in mCRC patients with MSI-H and $10 \%$ disease control in patients with MSS mCRC (36). Interestingly, their results revealed that a high tumor mutational burden (TMB) in mCRC may predict a better response, and nivolumab in combination with ipilimumab is a promising new therapeutic strategy for $\mathrm{dMMR} / \mathrm{MSI}-\mathrm{H}$ mCRC (36). Le et al. (42) also reported similar findings in CRC patients with different MSI statuses. The immune-related objective response rate (ORR) and the immune-related progression-free survival (PFS) rate to anti-PD-1 mAbs at primary endpoints of the study (20 weeks) were remarkedly higher in dMMR CRCs than that in PMMR CRC. Taken together, current studies with anti-PD-1 mAbs suggested that the therapeutic efficacy is generally unencouraging and response rates in patients with pMMR/MSI-L mCRC were seen only rarely. Thus, the dMMR 
status is an important factor in influencing therapeutic response of anti-PD-1 mAbs in treating patients with CRCs (43). Several reviews have summarized the distinct therapeutic response of anti-PD-1/PD-L1 mAbs in CRC patients with different MSI statuses; readers can refer to these articles.

In addition to anti-PD-1 mAbs, the therapeutic efficacy of anti-PD-L1 mAbs in mCRCs is also being evaluated. Brahmer et al. assessed the efficacy of an anti-human PD-L1 mAb in a cohort of more than 200 solid tumors that included 18 CRC patients. The results showed that none response was seen in patients with CRC (44). Furthermore, unlike its therapeutic efficacy demonstrated in other types of solid tumors such as advanced non-small cell lung cancer (45) and metastatic urothelial cancer (46), the response rate of atezolizumab (a humanized anti-PD-L1 mAb) in MSS CRCs is not encouraging (47). The therapeutic analysis of the combination of cobimetinib (a MEK protein kinase $\mathrm{mAb}$ ) with atezolizumab in 23 patients with mCRC suggests that the response rate was very low in CRC patients with pMMR/MSI-L (47).

For details, readers can refer to published literatures; several reviews have summarized such distinct therapeutic response of anti-PD-1/PD-L1 mAbs between dMMR/MSI-H CRCs and pMMR/MSI-L CRCs $(7,15,16,18,48-51)$. Further studies or trials should focus on how to improve therapeutic response rate of anti-PD-1/PD-L1 mAbs in patients with pMMR/MSI-L CRCs.

\section{THE POSSIBLE MECHANISMS LEADING TO A DISTINCT THERAPEUTIC EFFICACY OF ANTI-PD-1/PD-L1 MAbs BETWEEN dMMR/MSI-H CRCs AND PMMR/MSI-L CRCs}

Clinical studies and trials have shown that patients with dMMR/ MSI-H CRC and PMMR/MSI-L or MSS CRC respond inconsistently to anti-PD-1/PD-L1 mAbs, which is associated with differences in the components of TIME. Indeed, it has been shown that multiple factors existed in the TIME, including immune cell phenotypes, cytokine networks, and immune checkpoints, differ between dMMR/MSI-H CRCs and pMMR/ MSI-L CRCs (12, 15, 52-57) (see summarization in Table 1).

Studies have revealed that TMB in dMMR/MSI-H CRCs is significantly higher than that in pMMR/MSI-L CRCs $(48,67-$ 69), and an increased amount of TMB in dMMR/MSI-H tumours can result in a 20-time higher rate of mutation and a stronger immune response than pMMR/MSI-L tumours (52, 53, 58 ), which are reflected in the very different composition and function of immune infiltrates in the TIME (70-73). Therefore, TMB appears to be an important biomarker for patients with dMMR/MSI-H CRC in response to anti-PD-1 therapy $(68,74-$ 77). Schrock et al. reported that TMB was strongly associated with objective response and favorable progression-free survival, by univariate $(\mathrm{P}<0.001)$ and multivariate analysis in MSI-H mCRC patients treated with anti-PD-1/PD-L1 mAbs (68). Fotios Loupakis et al. reported that higher TMB and increased number of TILs in patients with dMMR/MSI-H CRCs were likely related to a better response to therapies of checkpoint inhibitors (78). Consequently, numerous studies demonstrated that increased densities of infiltrating immune cells i.e. TH9 subset may possibly contribute to the improved therapeutic response of dMMR/MSI-H CRCs to anti-PD-1/PD-L1 mAb treatment (59, 78-80). Zhang et al. (54) have immunohistochemically shown that the TIME of dMMR/MSI-H CRCs differs from that of pMMR/MSI-L CRCs. The density of PD-1 positive TILs was higher in CRC patients with dMMR/MSI-H than those with pMMR/MSI-L. The positive rate of PD-L1 on immune cells in the TIME also differed between dMMR/MSI-H CRCs and pMMR/MSI-L CRCs. In addition, the level of immunosuppressive factor indoleamine 2,3 dioxygenase (IDO) expression was greater in CRC patients with dMMR/MSI-H CRCs than those with pMMR/MSI-L $(65,66)$.

PD-L1 is the ligand for PD-1 and plays an important negative regulatory effect by binding $\mathrm{PD}-1$, resulting in the restricting of anti-tumor function of T lymphocytes $(81,82)$. Liu et al. (43) demonstrated that dMMR/MSI-H CRCs more frequently exhibited higher populations of TILs and PD-L1-positive cells than PMMR/MSI-L CRCs. Furthermore, PD-L1 expression in patients with CRC was associated with clinicopathologic and molecular features and a worse outcome in CRC patients with dMMR/MSI-H than those with pMMR/MSI-L (83). PD-L1 expression was closely related to dMMR/MSI-H status in patients with CRC. Taube et al. reported that PD-L1 expression was identified in $42.4 \%$ of dMMR/MSI-H CRCs with TILs and only $18.0 \%$ pMMR/MSI-L CRCs with TILs (43). They also found that tumor PD-L1 expression reflected an immune-active microenvironment, while it did so in association with other immunosuppressive molecules, including PD-1 and PD-L2, it was the single factor most closely correlated with response to anti-PD-1 mAbs in patients with melanoma, non-small cell lung carcinoma, renal cell carcinoma, CRC, or castration-resistant prostate cancer (57). Liu et al. (84) immunohistochemically examined the cellular localization of PD-L1 in 73 dMMR CRCs and 56 pMMR CRCs respectively. They found that the expression of PD-L1 was

TABLE 1 | Difference of tumor immune microenvironment (TIME) between $\mathrm{dMMR/MSI-H} \mathrm{CRCs} \mathrm{and} \mathrm{pMMR/MSI-L} \mathrm{CRCs.}$

Components (reference ID)

dMMR/MSI-H vs. pMMR/MSI-L

TIL (43)

Effector-memory T cells (58)

TH1 cells (58)

Macrophage (59)

DC (59)

Foxp3 low non-suppressive Tregs (60)

PD1/PD-L1 expression $(58,61,62)$

CTLA4 expression (5)

IFN- $\gamma$ expression $(25,63,64)$

IDO $(65,66)$

CTLA4, Cytotoxic T-lymphocyte-associated antigen 4, DC, dendritic cell; IDO, indolamine 2'3'-dioxygenase; IFN- $\gamma$, interferon- $\gamma, P D-1$, programmed death-1 receptor; $P D-1 L$, programmed death-1 receptor ligand; MSI-H, microsatellite-instability-high; MSI-L, microsatellite-instability-low; IMMR, mismatch repair-deficient; pMMR, mismatch repair-proficient; TIL, tumor infiltrating lymphocyte; Treg, regulatory $T$ cell. 
primarily observed in tumor infiltrating immune cells, particularly in immune cells at the site of invasive front with tumor-stroma-interface. The population level of immune cell positive for PD-L1 staining was significantly higher in dMMR tumors than in pMMR tumors (84). Both Gatalica and Inaguma have confirmed a similar result in CRC patients with different MSI statuses $(61,62)$. dMMR/MSI-H CRC patients with a higher PD-L1 level had a deeper functional suppression of TILs than those pMMR/MSI-L CRC patients with a lower PD-L1 level, and blocking PD-1/PD-L1 might relieve such suppression and reactivate the function of TILs $(61,62)$. Therefore, PD-L1 expression in diverse solid tumors might stratify patients in terms of response to anti-PD-1/PD-L1 immunotherapy and sensitivity to anti-PD-1/anti-PD-L1 mAbs (85), and PD-L1 expression was related to intrinsic and adaptive immune resistance in cancer patients receiving immunotherapy (86). Analysis of TCGA data has also revealed that the mRNA expression levels of both PD-L1 and PD-L2 were significantly upregulated in dMMR/MSI-H CRCs compared with pMMR/ MSI-L CRCs (87). Indeed, clinical studies identified that mCRC patients with higher PD-L1 expression in CRC cells might had a higher response rate than those patients with lower PD-L1 expression in CRC cells $(42,88)$, which was also supported by a meta-analysis of PD-L1 expression in patients with melanoma and lung and genitourinary cancers (89). However, Le Flahec et al.'s (90) immunohistochemistry compared PD-L1 expression in whole tumor specimens and tissue microarray slides between dMMR/MSI-H CRCs and pMMR/MSI-L CRCs. They reported no significant difference in PD-L1 expression levels between dMMR and pMMR CRCs. Thus, they concluded that the value of PD-L1 in explaining the different therapeutic response rate between $\mathrm{AMMR}$ CRCs and PMMR CRCs remains unclear, and further studies are required.

Taken together, PD-1 expression observed on the increased populations of immune cells in dMMR/MSI-H CRCs may act as a modulatory factor for the immune response. The use of anti-PD-1 mAbs blocks the expression of PD-1 on tumor cells and TILs and could further reactivate other types of immune cells and finally enhances host anti-tumor immune response in patients with $\mathrm{dMMR} / \mathrm{MSI}-\mathrm{H}$ CRC. Therefore, the response rate to anti-PD-1 and anti-PD-L1 mAbs in patients with $\mathrm{dMMR} / \mathrm{MSI}-\mathrm{H}$ CRC is higher than that in patients with pMMR/MSI-L mCRC.

Different levels of cytokines produced by both immune cells and tumor cells in the tumor microenvironment may also contribute to a distinct therapeutic response between dMMR/ MSI-H CRCs and pMMR/MSI-L CRCs. For example, IFN- $\gamma$ produced by activated $\mathrm{T}$ lymphocytes is an important modulator of PD-L1 expression, and it can significantly upregulate PD-L1 expression in both tumor cells and immune cells in the TIME $(69,86)$. Studies have shown that responders had elevated expression levels of IFN- $\gamma$ and IFN- $\gamma$-inducible genes prior anti-PD-L1 mAb treatment in patients with melanoma or renal cell carcinoma $(25,63,64)$. However, its role in influencing the response rate to anti-PD-1/PD-L1 mAb therapy in patients with $\mathrm{dMMR} / \mathrm{MSI}-\mathrm{H}$ CRC needs further investigation.
Finally, host anti-tumor immunity is regulated by a complex immune cellular network in patents with $\operatorname{CRC}(1,3,70,91-93)$. For instance, a number of recent studies have reported that diverse TH subsets TH9, TH17 and TH22 and their main cytokines such as IL-9, IL-17 and IL-22 participate in the modulation of immune response in patients with CRC (91). Immune scores between dMMR/MSI-H CRCs and pMMR/MSI-L or MSS CRCs were also very different $(94,95)$ in which, populations of CD3-positive and CD4-positive TILs in tissue microarray samples collected from the tumor center and invasive front were significantly higher in CRC patients with $\mathrm{dMMR} / \mathrm{MSI}-\mathrm{H}$ than those with $\mathrm{pMMR} / \mathrm{MSI}-\mathrm{L}$ (94). The phenotypes of infiltrating immune cells were also different between dMMR/MSI-H and pMMR/MSI-L CRCs (95). In addition to the infiltration of lymphocytes, many other cellular components of the CRC TIME might be different (96). For example, local immunosuppressive cells, including Tregs and myeloid-derived suppressor cells (MDSCs), were ordinarily observed in the CRC TIME $(97,98)$. Le Gouvello et al. reported remarkable increased levels of Foxp3-positive Tregs and IL-17 in CRC patients with pMMR/MSI-L (99). These cells might be involved in the determination of anti-PD-1 therapeutic efficacy in patients with CRC. One study conducted in the human hepatoma cell lines showed that Tregs potentially inhibited IFN- $\gamma$ secretion and the cytotoxicity of CD8-positive T cells (100). Moreover, Bauer $\mathrm{K}$ et al. (101) reported that the existence of Tregs greatly influenced the frequency of effector $\mathrm{T}$ cells in response to specific MSI-H-related frameshift peptides in CRC. Dyck and colleagues (102) reported that high PD-1 expression correlated with increased tumor-infiltrating Tregs, and reduced effector $\mathrm{T}$ cells and blocking PD-1 might effectively enhance antitumor immunity. Zhang et al. (103) have further shown that PD-1 mAbs could reverse Treg suppression. Toor et al. (21) demonstrated that Tregs might hamper the response to immune checkpoint blockade (anti-PD-1 mAb) in patients with $\mathrm{CRC}$, and the administration of an anti-PD-1 mAb in mice could significantly decrease tumor-infiltrating Tregs and increase TILs in the CRC TIME (104). Interestingly, a higher expression of FoxP3 (a marker of Tregs) has been demonstrated in patients with pMMR/MSI-L CRC than in those with dMMR/MSI-H CRC, indicating a deeper degree of immunosuppression in these patients. As a result, a higher proportion of Tregs is associated with a poor prognosis for immunotherapy, and the rate of therapeutic response to anti-PD-1 mAbs might be lower in patients with pMMR than that in patients with $\mathrm{dMMR}$. Therefore, eliciting an enhanced response to anti-PD-1/PD-L1 mAbs in patients with $\mathrm{pMMR} / \mathrm{MSI}-\mathrm{L}$ CRC may require combined therapeutics with other anti-immunosuppressive, antiangiogenic or anti-immune checkpoint bioagents $(42,105)$.

Thus, accumulating evidence suggests that multiple factors are involved in the lack of sensitivity to anti-PD1/PD-L1 mAb therapy seen in patients with pMMR/MSI-L or MSS tumours (see Figure 2). The above possible mechanisms might provide useful information for future improvements. For example, the host immune response to TMB was very different between patients with $\mathrm{dMMR} / \mathrm{MSI}-\mathrm{H}$ CRC and those with pMMR/MSI-L CRC $(76,94)$, and a single anti-PD-1/PD-L1 mAbs could not 
block all the regulatory pathways/signals. Recent studies revealed that the calcium/calmodulin-dependent protein kinase $1 \mathrm{D}$ (CAMK1D) and the m6A demethylase Alkbh5 may regulate the tumor cells refractory to anti-PD-L1 treatment $(106,107)$. However, the difference of the CAMK1D and m6A demethylase Alkbh5 expressions between dMMR/MSI-H and pMMR/MSI-L CRCs has not been well studied so far. Furthermore, a recent study suggested that transcriptional factor STAT was involved in the response to anti-PD-1 in patients with MSS CRCs (108). Finally, it is important to state that not all dMMR/MSI-H CRCs respond to immunotherapy. Combinations of anti-PD-1/PD-L1 $\mathrm{mAbs}$ with other anti-tumor agents may improve the therapeutic efficacy in patients with CRCs (69). Trials of atezolizumab in combination with both bevacizumab and cobimetinib mAbs have opened the way for combination strategies, which could extend the indication for immune checkpoint inhibitors to pMMR mCRC. Similarly, the combination of nivolumab and ipilimumab mAbs in dMMR/MSI-H mCRC patients has shown an encouraging clinical outcome (36).

\section{TRANSLATIONAL SIGNIFICANCE OF MECHANISMS LEADING TO DISTINCT OUTCOMES BETWEEN dMMR/MSI-H CRCs AND pMMR/MSI-L CRCs}

Accumulative evidence has suggested that multiple factors such as $\mathrm{TMB}$, expression levels of $\mathrm{PD}-1 / \mathrm{PD}-\mathrm{L} 1$ and cytokines and

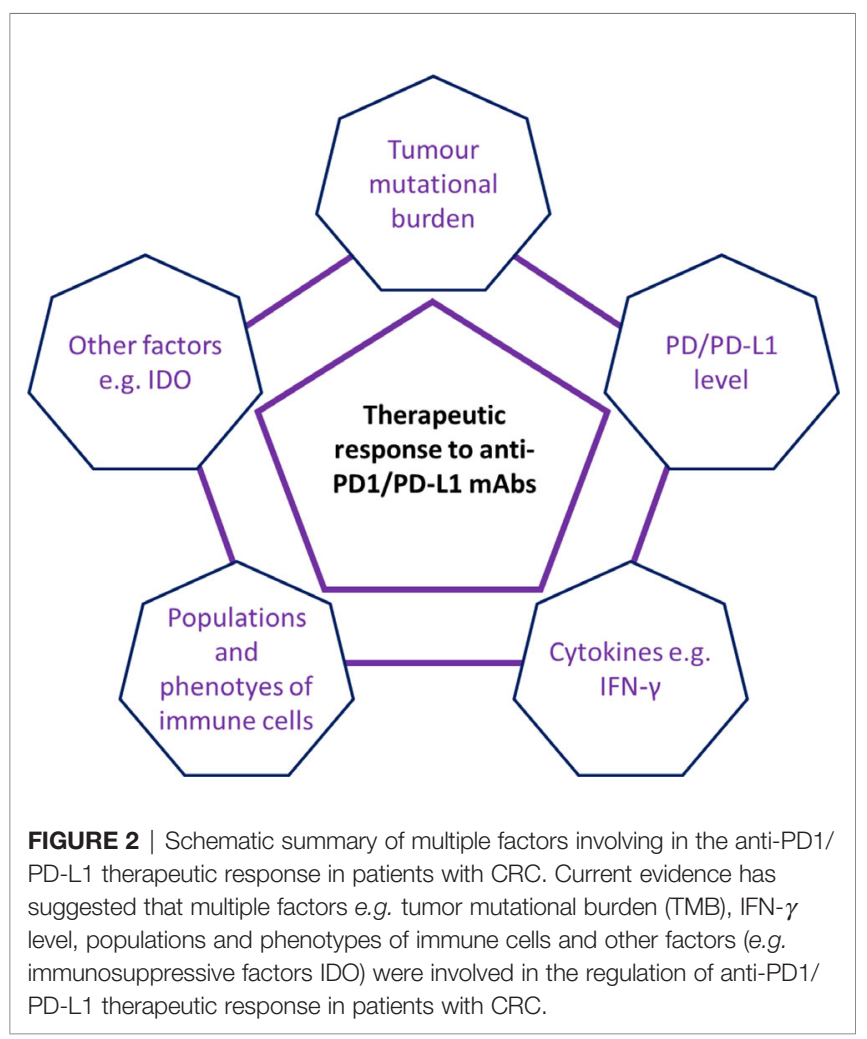

immune infiltrates are involved in the mechanisms of distinct response to anti-PD-1/PD-L1 mAbs between dMMR/MSI-H CRCs and pMMR/MSI-L CRCs. The discovery of potential mechanisms leading to distinct therapeutic outcomes of antiPD-1/PD-L1 therapy seen between dMMR/MSI-H CRCs and pMMR/MSI-L CRCs might have an important translational significance $(55,109)$.

Firstly, the study of potential mechanisms leading to a distinct therapeutic efficacy of PD-1/PD-L1 inhibitors seen between $\mathrm{dMMR} / \mathrm{MSI}-\mathrm{H}$ and $\mathrm{pMMR} / \mathrm{MSI}-\mathrm{L}$ CRCs might help clinicians to select candidates for anti-PD-1/PD-L1 therapy. Anti-PD-1/ PD-L1 therapy is an expensive biotherapeutic. Treating cost studies have revealed that treating with immune checkpoint inhibitors may averagely cost $\$ 1$ million per patient (110). By analyzing relative factors, clinicians can identify candidates (CRC patients) who will benefit from anti-PD-1/PD-L1 therapy. For example, studies have reported that mCRC patients with higher PD-L1 expression might have a higher response rate than those patients with lower PD-L1 expression $(42,88)$. Furthermore, it may help clinicians to select reliable biomarker in the evaluation of anti-PD-1/PD-L1 therapeutic response in patients with CRC. Clinicians can measure the expression level of PD-L1 in tumor cells and immune cells examined by diverse techniques i.e. immunohistochemistry and in situ hybridization, and in whole tissue specimens measured by real-time PCR to provide important predicating information of response prior treatments. To improve and optimize the efficacy of anti-immune checkpoint therapies, a combination of anti-PD-1/PD-L mAbs with other anti-tumor bioagents probably being the most relevant strategy $(36,111$, 112). For instance, several studies showed that IFN- $\gamma$ can significantly upregulate PD-L1 expression in both tumor cells and immune cells $(69,86,113)$. This finding raises an interesting possibility that whether combination biotherapy of anti-PD-1/ PD-L1 mAbs with IFN- $\gamma$ can induce an enhanced response rate in patients with CRC should be tested. Moreover, an improved therapeutic efficacy has been demonstrated in cancer-favoring oncolytic vaccinia virus combined with anti-PD-1 in treating mouse CRC (106). Cai et al. (114) reported an upregulated effect of angiogenesis inhibitor apatinib on the expression of PD-1 at mRNA and protein levels but a downregulated effect on IFN- $\gamma$ secretion from $\mathrm{T}$ cells in various colon cancer cells. The combination of apatinib with anti-PD-1 antibody could result in a better efficacy in a syngeneic mouse model (CT-26 cells in $\mathrm{Balb} / \mathrm{c}$ ) than single-drug treatment. More recently, Knudson et al. showed that IL-15 super-agonist N-803 plus anti-PD-L1 $\mathrm{mAb}$ could reduce MC38-CEA (colon cancer cell) tumor burden and increased survival rate as compared to N-803 and anti-PDL1 monotherapies in MC38-CEA colon tumor-bearing mice (115). An enhanced therapeutic effect of F8-IL2 (an antibodyIL2 fusion protein) bombinated with anti-PD-1, anti-PD-L1 and anti-CTLA-4 antibodies was observed in immunocompetent mice bearing CT26 tumours (116). These studies may provide a rationale for the combination of anti-PD-1 antibody with other anti-tumor agents for CRC treatment in the clinic. Finally, as the mechanisms responsible for distinct responses to PD-1/PD-L1 


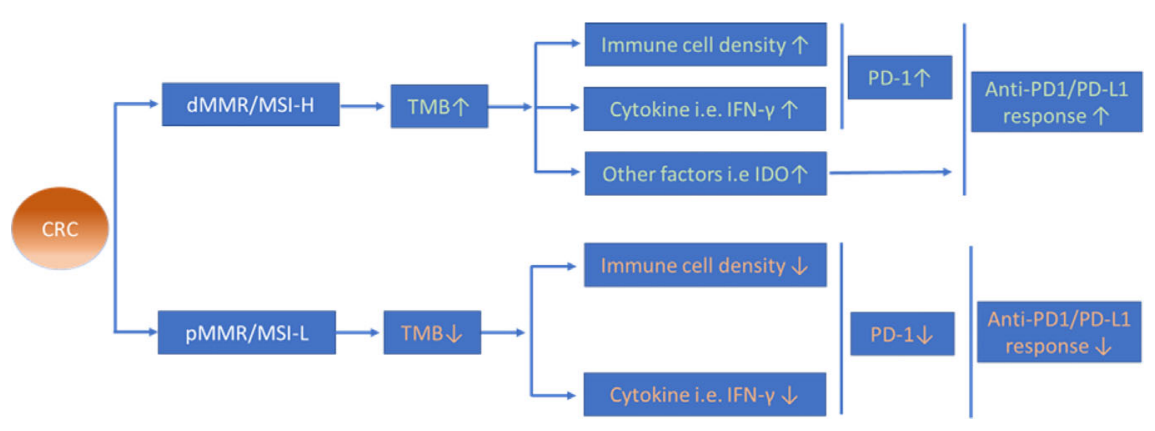

FIGURE 3 | Possible mechanisms of different anti-PD-1/PD-L1 therapeutic efficacy between CRC with dMMR/MSI-H and pMMR/MSI-L. Increased TMB in dMMR/ MSI-H CRCs is proposed to induce higher infiltration of immune cells and expression level of cytokines in the tumour microenvironment that might contribute to the distinct therapeutic efficacy of anti-PD-1/PD-L1 therapy between dMMR/MSI-H CRCs and PMMR/MSI-L CRCs.

mAbs in patients with CRC are elucidated, how to convert a 'nonimmunogenic' CRC into an 'immunogenic' CRC becomes a critical important issue $(18,117)$. Wang et al. recently investigated the efficacy of fruquintinib (a novel anti-vascular endothelial growth factor receptor tyrosine kinase inhibitor) plus anti-PD-1 mAb for MSS CRC in a murine syngeneic model of CT26 cells and verified that cotreatment significantly inhibited tumor growth and promoted survival time for tumor-bearing mice compared with the single drug alone (105).

\section{CONCLUDING REMARKS}

Division of mCRCs into dMMR/MSI-H and pMMR/MSI-L subsets yields a distinct therapeutic response to anti-PD-1/PDL1 immunotherapies, in which dMMR/MSI-H CRCs seem to be relatively more "sensitive" in response to anti-PD-1/PD-L1 mAbs than those CRCs with pMMR/MSI-L. The possible mechanisms leading to such distinct outcomes may relate to multiple factors e.g. TMB, TILs, and immunosuppressive cells, the expression level of PD-L1 and the complex cytokine network

\section{REFERENCES}

1. Cui G, Qi H, Gundersen MD, Yang H, Christiansen I, Sorbye SW, et al. Dynamics of the IL-33/ST2 network in the progression of human colorectal adenoma to sporadic colorectal cancer. Cancer Immunol Immunother (2015) 64(2):181-90. doi: 10.1007/s00262-014-1624-x

2. Cui G, Yuan A, Goll R, Florholmen J. IL-17A in the tumor microenvironment of the human colorectal adenoma-carcinoma sequence. Scand J Gastroenterol (2012) 47(11):1304-12. doi: 10.3109/ 00365521.2012 .725089

3. Cui G, Yuan A, Pang Z, Zheng W, Li Z, Goll R. Contribution of IL-33 to the Pathogenesis of Colorectal Cancer. Front Oncol (2018) 8:561. doi: 10.3389/ fonc.2018.00561

4. Cui G, Yuan A, Sun Z, Zheng W, Pang Z. IL-1beta/IL-6 network in the tumor microenvironment of human colorectal cancer. Pathol Res Pract (2018) 214(7):986-92. doi: 10.1016/j.prp.2018.05.011

5. Llosa NJ, Cruise M, Tam A, Wicks EC, Hechenbleikner EM, Taube JM, et al. The vigorous immune microenvironment of microsatellite instable colon cancer is balanced by multiple counter-inhibitory checkpoints. Cancer Discovery (2015) 5(1):43-51. doi: 10.1158/2159-8290.CD-14-0863 in the CRC microenvironment (see Figure 3). Future studies should discover strategies that determine how to convert a 'nonimmunogenic' CRC into an 'immunogenic' CRC and improve the therapeutic response rate to anti-PD-1/PD-L1 mAbs in patients with pMMR/MSI-L mCRC and the optimal strategy for identifying CRC patients who will benefit from anti-PD-1/ $\mathrm{PD}-\mathrm{L} 1 \mathrm{mAb}$ therapy prior treatments in the clinic.

\section{AUTHOR CONTRIBUTIONS}

The author confirms being the sole contributor of this work and has approved it for publication.

\section{FUNDING}

This study was supported by the National Nature Science Foundation of China (Program No. 81071969) and the Medical Research Program, Northern Norway Regional Health Authority, Norway (Program No. SFP-44-04).

6. Marisa L, Svrcek M, Collura A, Becht E, Cervera P, Wanherdrick K, et al The Balance Between Cytotoxic T-cell Lymphocytes and Immune Checkpoint Expression in the Prognosis of Colon Tumors. J Natl Cancer Inst (2018) 110(1):68-77. doi: 10.1093/jnci/djx136

7. Passardi A, Canale M, Valgiusti M, Ulivi P. Immune Checkpoints as a Target for Colorectal Cancer Treatment. Int J Mol Sci (2017) 18(6):13. doi: 10.3390/ijms18061324

8. Mahoney KM, Freeman GJ, McDermott DF. The Next Immune-Checkpoint Inhibitors: PD-1/PD-L1 Blockade in Melanoma. Clin Ther (2015) 37 (4):764-82. doi: 10.1016/j.clinthera.2015.02.018

9. Motzer RJ, Tannir NM, McDermott DF, Aren Frontera O, Melichar B, Choueiri TK, et al. Nivolumab plus Ipilimumab versus Sunitinib in Advanced Renal-Cell Carcinoma. N Engl J Med (2018) 378(14):1277-90. doi: 10.1056/NEJMoa1712126

10. Chen YM. Immune checkpoint inhibitors for nonsmall cell lung cancer treatment. J Chin Med Assoc (2017) 80(1):7-14. doi: 10.1016/j.jcma.2016.08.005

11. Toh JWT, de Souza P, Lim SH, Singh P, Chua W, Ng W, et al. The Potentia Value of Immunotherapy in Colorectal Cancers: Review of the Evidence for Programmed Death-1 Inhibitor Therapy. Clin Colorectal Cancer (2016) 15 (4):285-91. doi: 10.1016/j.clcc.2016.07.007 
12. Marginean EC, Melosky B. Is There a Role for Programmed Death Ligand-1 Testing and Immunotherapy in Colorectal Cancer With Microsatellite Instability? Part I-Colorectal Cancer: Microsatellite Instability, Testing, and Clinical Implications. Arch Pathol Lab Med (2018) 142(1):17-25. doi: 10.5858/arpa.2017-0040-RA

13. Zhang CM, Lv JF, Gong L, Yu LY, Chen XP, Zhou HH, et al. Role of Deficient Mismatch Repair in the Personalized Management of Colorectal Cancer. Int J Environ Res Public Health (2016) 13(9). doi: 10.3390/ ijerph13090892

14. Borcherding N, Kolb R, Gullicksrud J, Vikas P, Zhu Y, Zhang W. Keeping Tumors in Check: A Mechanistic Review of Clinical Response and Resistance to Immune Checkpoint Blockade in Cancer. J Mol Biol (2018) 430(14):2014-29. doi: 10.1016/j.jmb.2018.05.030

15. Marginean EC, Melosky B. Is There a Role for Programmed Death Ligand-1 Testing and Immunotherapy in Colorectal Cancer With Microsatellite Instability? Part II-The Challenge of Programmed Death Ligand-1 Testing and Its Role in Microsatellite Instability-High Colorectal Cancer. Arch Pathol Lab Med (2018) 142(1):26-34. doi: 10.5858/arpa.2017-0041-RA

16. Yaghoubi N, Soltani A, Ghazvini K, Hassanian SM, Hashemy SI. PD-1/ PDL1 blockade as a novel treatment for colorectal cancer. BioMed Pharmacother (2019) 110:312-8. doi: 10.1016/j.biopha.2018.11.105

17. Hewish M, Lord CJ, Martin SA, Cunningham D, Ashworth A. Mismatch repair deficient colorectal cancer in the era of personalized treatment. Nat Rev Clin Oncol (2010) 7(4):197-208. doi: 10.1038/nrclinonc.2010.18

18. Ganesh K, Stadler ZK, Cercek A, Mendelsohn RB, Shia J, Segal NH, et al. Immunotherapy in colorectal cancer: rationale, challenges and potential. Nat Rev Gastroenterol Hepatol (2019) 16(6):361-75. doi: 10.1038/s41575-0190126-x

19. Dudley JC, Lin MT, Le DT, Eshleman JR. Microsatellite Instability as a Biomarker for PD-1 Blockade. Clin Cancer Res (2016) 22(4):813-20. doi: 10.1158/1078-0432.CCR-15-1678

20. Postow MA, Callahan MK, Wolchok JD. Immune Checkpoint Blockade in Cancer Therapy. J Clin Oncol (2015) 33(17):1974-82. doi: 10.1200/ JCO.2014.59.4358

21. Toor SM, Murshed K, Al-Dhaheri M, Khawar M, Abu Nada M, Elkord E. Immune Checkpoints in Circulating and Tumor-Infiltrating CD4(+) T Cell Subsets in Colorectal Cancer Patients. Front Immunol (2019) 10:2936. doi: 10.3389/fimmu.2019.02936

22. Kim JW, Eder JP. Prospects for targeting PD-1 and PD-L1 in various tumor types. Oncol (Williston Park) (2014) 28 Suppl 3:15-28.

23. Topalian SL, Taube JM, Anders RA, Pardoll DM. Mechanism-driven biomarkers to guide immune checkpoint blockade in cancer therapy. Nat Rev Cancer (2016) 16(5):275-87. doi: 10.1038/nrc.2016.36

24. Zaravinos A, Roufas C, Nagara M, de Lucas Moreno B, Oblovatskaya M, Efstathiades $\mathrm{C}$, et al. Cytolytic activity correlates with the mutational burden and deregulated expression of immune checkpoints in colorectal cancer. J Exp Clin Cancer Res (2019) 38(1):364. doi: 10.1186/s13046-019-1372-z

25. Buder-Bakhaya K, Hassel JC. Biomarkers for Clinical Benefit of Immune Checkpoint Inhibitor Treatment-A Review From the Melanoma Perspective and Beyond. Front Immunol (2018) 9:1474. doi: 10.3389/fimmu.2018.01474

26. Krummel MF, Allison JP. CTLA-4 engagement inhibits IL-2 accumulation and cell cycle progression upon activation of resting T cells. J Exp Med (1996) 183(6):2533-40. doi: 10.1084/jem.183.6.2533

27. Funt SA, Page DB, Wolchok JD, Postow MA. CTLA-4 antibodies: new directions, new combinations. Oncol (Williston Park) (2014) 28 Suppl 3:6-14.

28. Simpson TR, Li F, Montalvo-Ortiz W, Sepulveda MA, Bergerhoff K, Arce F, et al. Fc-dependent depletion of tumor-infiltrating regulatory $\mathrm{T}$ cells codefines the efficacy of anti-CTLA-4 therapy against melanoma. J Exp Med (2013) 210(9):1695-710. doi: 10.1084/jem.20130579

29. Simeone E, Ascierto PA. Immunomodulating antibodies in the treatment of metastatic melanoma: the experience with anti-CTLA-4, anti-CD137, and anti-PD1. J Immunotoxicol (2012) 9(3):241-7. doi: 10.3109/15476 91X.2012.678021

30. Brahmer JR, Drake CG, Wollner I, Powderly JD, Picus J, Sharfman WH, et al. Phase I study of single-agent anti-programmed death-1 (MDX-1106) in refractory solid tumors: safety, clinical activity, pharmacodynamics, and immunologic correlates. J Clin Oncol (2010) 28(19):3167-75. doi: 10.1200/ JCO.2009.26.7609
31. Popat S, Hubner R, Houlston RS. Systematic review of microsatellite instability and colorectal cancer prognosis. J Clin Oncol (2005) 23(3):60918. doi: 10.1200/JCO.2005.01.086

32. Wu M, Kim YS, Ryu HS, Choi SC, Kim KY, Park WC, et al. MSI status is associated with distinct clinicopathological features in BRAF mutation colorectal cancer: A systematic review and meta-analysis. Pathol Res Pract (2020) 216(1):152791. doi: 10.1016/j.prp.2019.152791

33. Samowitz WS, Curtin K, Ma KN, Schaffer D, Coleman LW, Leppert M, et al. Microsatellite instability in sporadic colon cancer is associated with an improved prognosis at the population level. Cancer Epidemiol Biomarkers Prev (2001) 10(9):917-23.

34. Colle R, Cohen R, Cochereau D, Duval A, Lascols O, Lopez-Trabada D, et al. Immunotherapy and patients treated for cancer with microsatellite instability. Bull Cancer (2017) 104(1):42-51. doi: 10.1016/j. bulcan.2016.11.006

35. Naboush A, Roman CA, Shapira I. Immune checkpoint inhibitors in malignancies with mismatch repair deficiency: a review of the state of the current knowledge. J Investig Med (2017) 65(4):754-8. doi: 10.1136/jim2016-000342

36. Overman MJ, Lonardi S, Wong KYM, Lenz HJ, Gelsomino F, Aglietta M, et al. Durable Clinical Benefit With Nivolumab Plus Ipilimumab in DNA Mismatch Repair-Deficient/Microsatellite Instability-High Metastatic Colorectal Cancer. J Clin Oncol (2018) 36(8):773-9. doi: 10.1200/ JCO.2017.76.9901

37. Patnaik A, Kang SP, Rasco D, Papadopoulos KP, Elassaiss-Schaap J, Beeram M, et al. Phase I Study of Pembrolizumab (MK-3475; Anti-PD-1 Monoclonal Antibody) in Patients with Advanced Solid Tumors. Clin Cancer Res (2015) 21(19):4286-93. doi: 10.1158/1078-0432.CCR-14-2607

38. Topalian SL, Hodi FS, Brahmer JR, Gettinger SN, Smith DC, McDermott DF, et al. Safety, activity, and immune correlates of anti-PD-1 antibody in cancer. N Engl J Med (2012) 366(26):2443-54. doi: 10.1056/NEJMoa1200690

39. Yarchoan M, Hopkins A, Jaffee EM. Tumor Mutational Burden and Response Rate to PD-1 Inhibition. N Engl J Med (2017) 377(25):2500-1. doi: 10.1056/NEJMc1713444

40. Gong J, Wang C, Lee PP, Chu P, Fakih M. Response to PD-1 Blockade in Microsatellite Stable Metastatic Colorectal Cancer Harboring a POLE Mutation. J Natl Compr Canc Netw (2017) 15(2):142-7. doi: 10.6004/ jnccn.2017.0016

41. O’Neil BH, Wallmark JM, Lorente D, Elez E, Raimbourg J, Gomez-Roca C, et al. Safety and antitumor activity of the anti-PD-1 antibody pembrolizumab in patients with advanced colorectal carcinoma. PloS One (2017) 12(12):e0189848. doi: 10.1371/journal.pone.0189848

42. Le DT, Uram JN, Wang H, Bartlett BR, Kemberling H, Eyring AD, et al. PD1 Blockade in Tumors with Mismatch-Repair Deficiency. $N$ Engl J Med (2015) 372(26):2509-20. doi: 10.1056/NEJMoa1500596

43. Liu S, Kong P, Wang X, Yang L, Jiang C, He W, et al. Tumor microenvironment classification based on T-cell infiltration and PD-L1 in patients with mismatch repair-proficient and -deficient colorectal cancer. Oncol Lett (2019) 17(2):2335-43. doi: 10.3892/ol.2018.9826

44. Brahmer JR, Tykodi SS, Chow LQ, Hwu WJ, Topalian SL, Hwu P, et al. Safety and activity of anti-PD-L1 antibody in patients with advanced cancer. N Engl J Med (2012) 366(26):2455-65. doi: 10.1056/NEJMoa1200694

45. Lang D, Huemer F, Rinnerthaler G, Horner A, Wass R, Brehm E, et al. Therapy Line and Associated Predictors of Response to PD-1/PD-L1Inhibitor Monotherapy in Advanced Non-small-Cell Lung Cancer: A Retrospective Bi-centric Cohort Study. Targ Oncol (2019) 14(6):707-17. doi: 10.1007/s11523-019-00679-9

46. Petrylak DP, Powles T, Bellmunt J, Braiteh F, Loriot Y, Morales-Barrera R, et al. Atezolizumab (MPDL3280A) Monotherapy for Patients With Metastatic Urothelial Cancer: Long-term Outcomes From a Phase 1 Study. JAMA Oncol (2018) 4(4):537-44. doi: 10.1001/jamaoncol.2017.5440

47. Bendell JC, Kim TW, Goh BC, Wallin J, Oh D-Y, Han S-W, et al. Clinical activity and safety of cobimetinib (Cobi) and atezolizumab in colorectal cancer (CRC). J Clin Oncol (2016) 15 suppl:3502. doi: 10.1200/ JCO.2016.34.15_suppl.3502

48. Ciardiello D, Vitiello PP, Cardone C, Martini G, Troiani T, Martinelli E, et al. Immunotherapy of colorectal cancer: Challenges for therapeutic efficacy. Cancer Treat Rev (2019) 76:22-32. doi: 10.1016/j.ctrv.2019.04.003 
49. Llosa NJ, Luber B, Siegel N, Awan AH, Oke T, Zhu Q, et al. Immunopathologic Stratification of Colorectal Cancer for Checkpoint Blockade Immunotherapy. Cancer Immunol Res (2019) 7(10):1574-9. doi: 10.1158/2326-6066.CIR-18-0927

50. Payandeh Z, Khalili S, Somi MH, Mard-Soltani M, Baghbanzadeh A, Hajiasgharzadeh $\mathrm{K}$, et al. PD-1/PD-L1-dependent immune response in colorectal cancer. J Cell Physiol (2020) 235:5461-75. doi: 10.1002/jcp.29494

51. Oliveira AF, Bretes L, Furtado I. Review of PD-1/PD-L1 Inhibitors in Metastatic dMMR/MSI-H Colorectal Cancer. Front Oncol (2019) 9:396. doi: $10.3389 /$ fonc. 2019.00396

52. Becht E, de Reynies A, Giraldo NA, Pilati C, Buttard B, Lacroix L, et al. Immune and Stromal Classification of Colorectal Cancer Is Associated with Molecular Subtypes and Relevant for Precision Immunotherapy. Clin Cancer Res (2016) 22(16):4057-66. doi: 10.1158/1078-0432.CCR-15-2879

53. Narayanan S, Kawaguchi T, Peng X, Qi Q, Liu S, Yan L, et al. Tumor Infiltrating Lymphocytes and Macrophages Improve Survival in Microsatellite Unstable Colorectal Cancer. Sci Rep (2019) 9(1):13455. doi: 10.1038/s41598-019-49878-4

54. Zhang Y, Sun Z, Mao X, Wu H, Luo F, Wu X, et al. Impact of mismatchrepair deficiency on the colorectal cancer immune microenvironment. Oncotarget (2017) 8(49):85526-36. doi: 10.18632/oncotarget.20241

55. Nowicki TS, Hu-Lieskovan S, Ribas A. Mechanisms of Resistance to PD-1 and PD-L1 Blockade. Cancer J (2018) 24(1):47-53. doi: 10.1097/ PPO.0000000000000303

56. Wang C, Gong J, Tu TY, Lee PP, Fakih M. Immune profiling of microsatellite instability-high and polymerase epsilon (POLE)-mutated metastatic colorectal tumors identifies predictors of response to anti-PD-1 therapy. J Gastrointest Oncol (2018) 9(3):404-15. doi: 10.21037/jgo. 2018.01.09

57. Taube JM, Klein A, Brahmer JR, Xu H, Pan X, Kim JH, et al. Association of PD-1, PD-1 ligands, and other features of the tumor immune microenvironment with response to anti-PD-1 therapy. Clin Cancer Res (2014) 20(19):5064-74. doi: 10.1158/1078-0432.CCR-13-3271

58. Mlecnik B, Bindea G, Angell HK, Maby P, Angelova M, Tougeron D, et al. Integrative Analyses of Colorectal Cancer Show Immunoscore Is a Stronger Predictor of Patient Survival Than Microsatellite Instability. Immunity (2016) 44(3):698-711. doi: 10.1016/j.immuni.2016.02.025

59. Bauer K, Michel S, Reuschenbach M, Nelius N, von Knebel Doeberitz M, Kloor M. Dendritic cell and macrophage infiltration in microsatelliteunstable and microsatellite-stable colorectal cancer. Fam Cancer (2011) 10 (3):557-65. doi: 10.1007/s10689-011-9449-7

60. Hamada T, Zhang X, Mima K, Bullman S, Sukawa Y, Nowak JA, et al. Fusobacterium nucleatum in Colorectal Cancer Relates to Immune Response Differentially by Tumor Microsatellite Instability Status. Cancer Immunol Res (2018) 6(11):1327-36. doi: 10.1158/2326-6066.CIR-18-0174

61. Rizvi NA, Hellmann MD, Snyder A, Kvistborg P, Makarov V, Havel JJ, et al. Cancer immunology. Mutational landscape determines sensitivity to PD-1 blockade in non-small cell lung cancer. Science (2015) 348(6230):124-8. doi: $10.1126 /$ science.aaa1348

62. Gatalica Z, Snyder C, Maney T, Ghazalpour A, Holterman DA, Xiao N, et al. Programmed cell death 1 (PD-1) and its ligand (PD-L1) in common cancers and their correlation with molecular cancer type. Cancer Epidemiol Biomarkers Prev (2014) 23(12):2965-70. doi: 10.1158/1055-9965.EPI-140654

63. Herbst RS, Soria JC, Kowanetz M, Fine GD, Hamid O, Gordon MS, et al. Predictive correlates of response to the anti-PD-L1 antibody MPDL3280A in cancer patients. Nature (2014) 515(7528):563-7. doi: 10.1038/nature14011

64. Vento J, Mulgaonkar A, Woolford L, Nham K, Christie A, Bagrodia A, et al. PD-L1 detection using (89)Zr-atezolizumab immuno-PET in renal cell carcinoma tumorgrafts from a patient with favorable nivolumab response. J Immunother Cancer (2019) 7(1):144. doi: 10.1186/s40425-019-0607-z

65. Loupakis F, Maddalena G, Depetris I, Murgioni S, Bergamo F, Dei Tos AP, et al. Treatment with checkpoint inhibitors in a metastatic colorectal cancer patient with molecular and immunohistochemical heterogeneity in MSI/ dMMR status. J Immunother Cancer (2019) 7(1):297. doi: 10.1186/s40425019-0788-5

66. Lee SJ, Jun SY, Lee IH, Kang BW, Park SY, Kim HJ, et al. CD274, LAG3, and IDO1 expressions in tumor-infiltrating immune cells as prognostic biomarker for patients with MSI-high colon cancer. J Cancer Res Clin Oncol (2018) 144(6):1005-14. doi: 10.1007/s00432-018-2620-x

67. Kerepesi C, Bakacs T, Moss RW, Slavin S, Anderson CC. Significant association between tumor mutational burden and immune-related adverse events during immune checkpoint inhibition therapies. Cancer Immunol Immunother (2020) 69(5):683-7. doi: 10.1007/s00262-02002543-6

68. Schrock AB, Ouyang C, Sandhu J, Sokol E, Jin D, Ross JS, et al. Tumor mutational burden is predictive of response to immune checkpoint inhibitors in MSI-high metastatic colorectal cancer. Ann Oncol (2019) 30 (7):1096-103. doi: 10.1093/annonc/mdz134

69. Quiroga D, Lyerly HK, Morse MA. Deficient Mismatch Repair and the Role of Immunotherapy in Metastatic Colorectal Cancer. Curr Treat Options Oncol (2016) 17(8):41. doi: 10.1007/s11864-016-0414-4

70. Shi Y, Li Z, Zheng W, Liu X, Sun C, Laugsand JB, et al. Changes of immunocytic phenotypes and functions from human colorectal adenomatous stage to cancerous stage: Update. Immunobiology (2015) 220 (10):1186-96. doi: 10.1016/j.imbio.2015.06.003

71. Ferris RL, Galon J. Additional Support for the Introduction of Immune Cell Quantification in Colorectal Cancer Classification. J Natl Cancer Inst (2016) 108:djw 033. doi: 10.1093/jnci/djw033

72. Galon J, Costes A, Sanchez-Cabo F, Kirilovsky A, Mlecnik B, Lagorce-Pages C, et al. Type, density, and location of immune cells within human colorectal tumors predict clinical outcome. Science (2006) 313(5795):1960-4. doi: 10.1126/science.1129139

73. Calon A, Lonardo E, Berenguer-Llergo A, Espinet E, HernandoMomblona X, Iglesias $\mathrm{M}$, et al. Stromal gene expression defines poorprognosis subtypes in colorectal cancer. Nat Genet (2015) 47(4):320-9. doi: $10.1038 /$ ng. 3225

74. Damilakis E, Mavroudis D, Sfakianaki M, Souglakos J. Immunotherapy in Metastatic Colorectal Cancer: Could the Latest Developments Hold the Key to Improving Patient Survival? Cancers (Basel) (2020) 12(4). doi: 10.3390/ cancers 12040889

75. Fabrizio DA, George TJJr., Dunne RF, Frampton G, Sun J, Gowen K, et al. Beyond microsatellite testing: assessment of tumor mutational burden identifies subsets of colorectal cancer who may respond to immune checkpoint inhibition. J Gastrointest Oncol (2018) 9(4):610-7. doi: 10.21037/ jgo.2018.05.06

76. Nebot-Bral L, Coutzac C, Kannouche PL, Chaput N. Why is immunotherapy effective (or not) in patients with MSI/MMRD tumors? Bull Cancer (2019) 106(2):105-13. doi: 10.1016/j.bulcan.2018.08.007

77. Mutation Burden Predicts Anti-PD-1 Response. Cancer Discovery (2018) 8 (3):258. doi: 10.1158/2159-8290.CD-NB2018-005

78. Loupakis F, Depetris I, Biason P, Intini R, Prete AA, Leone F, et al. Prediction of Benefit from Checkpoint Inhibitors in Mismatch Repair Deficient Metastatic Colorectal Cancer: Role of Tumor Infiltrating Lymphocytes. Oncologist (2020) 25(6):481-7. doi: 10.1634/theoncologist.2019-0611

79. Wang C, Lu Y, Chen L, Gao T, Yang Q, Zhu C, et al. Th9 cells are subjected to PD-1/PD-L1-mediated inhibition and are capable of promoting CD8 T cell expansion through IL-9R in colorectal cancer. Int Immunopharmacol (2020) 78:106019. doi: 10.1016/j.intimp.2019.106019

80. Limagne E, Euvrard R, Thibaudin M, Rebe C, Derangere V, Chevriaux A, et al. Accumulation of MDSC and Th17 Cells in Patients with Metastatic Colorectal Cancer Predicts the Efficacy of a FOLFOX-Bevacizumab Drug Treatment Regimen. Cancer Res (2016) 76(18):5241-52. doi: 10.1158/00085472.CAN-15-3164

81. Dong H, Zhu G, Tamada K, Chen L. B7-H1, a third member of the B7 family, co-stimulates T-cell proliferation and interleukin-10 secretion. Nat Med (1999) 5(12):1365-9. doi: 10.1038/70932

82. Butte MJ, Keir ME, Phamduy TB, Sharpe AH, Freeman GJ. Programmed death-1 ligand 1 interacts specifically with the B7-1 costimulatory molecule to inhibit T cell responses. Immunity (2007) 27(1):111-22. doi: 10.1016/ j.immuni.2007.05.016

83. Rosenbaum MW, Bledsoe JR, Morales-Oyarvide V, Huynh TG, MinoKenudson M. PD-L1 expression in colorectal cancer is associated with microsatellite instability, BRAF mutation, medullary morphology and cytotoxic tumor-infiltrating lymphocytes. Mod Pathol (2016) 29(9):110412. doi: $10.1038 /$ modpathol.2016.95 
84. Liu S, Gnen M, Stadler ZK, Weiser MR, Hechtman JF, Vakiani E, et al. Cellular localization of PD-L1 expression in mismatch-repair-deficient and proficient colorectal carcinomas. Mod Pathol (2019) 32(1):110-21. doi: 10.1038/s41379-018-0114-7

85. Noguchi T, Ward JP, Gubin MM, Arthur CD, Lee SH, Hundal J, et al. Temporally Distinct PD-L1 Expression by Tumor and Host Cells Contributes to Immune Escape. Cancer Immunol Res (2017) 5(2):106-17. doi: 10.1158/2326-6066.CIR-16-0391

86. Topalian SL, Drake CG, Pardoll DM. Immune checkpoint blockade: a common denominator approach to cancer therapy. Cancer Cell (2015) 27 (4):450-61. doi: 10.1016/j.ccell.2015.03.001

87. Kikuchi T, Mimura K, Okayama H, Nakayama Y, Saito K, Yamada L, et al. A subset of patients with MSS/MSI-low-colorectal cancer showed increased CD8(+) TILs together with up-regulated IFN-gamma. Oncol Lett (2019) 18 (6):5977-85. doi: 10.3892/ol.2019.10953

88. Meng X, Huang Z, Teng F, Xing L, Yu J. Predictive biomarkers in PD-1/PDL1 checkpoint blockade immunotherapy. Cancer Treat Rev (2015) 41 (10):868-76. doi: 10.1016/j.ctrv.2015.11.001

89. Carbognin L, Pilotto S, Milella M, Vaccaro V, Brunelli M, Calio A, et al. Differential Activity of Nivolumab, Pembrolizumab and MPDL3280A according to the Tumor Expression of Programmed Death-Ligand-1 (PD-L1): Sensitivity Analysis of Trials in Melanoma, Lung and Genitourinary Cancers. PloS One (2015) 10(6):e0130142. doi: 10.1371/journal.pone.0130142

90. Le Flahec G, Badic B, Guibourg B, Doucet L, Bail JP, Marcorelles P, et al. Mismatch repair-deficient colorectal cancer: a model of immunogenic and immune cell-rich tumor despite nonsignificant programmed cell death ligand-1 expression in tumor cells. Hum Pathol (2018) 72:135-43. doi: 10.1016/j.humpath.2017.09.019

91. Cui G. TH9, TH17, and TH22 Cell Subsets and Their Main Cytokine Products in the Pathogenesis of Colorectal Cancer. Front Oncol (2019) 9:1002. doi: 10.3389/fonc.2019.01002

92. Hua W, Yuan A, Zheng W, Li C, Cui J, Pang Z, et al. Accumulation of FoxP3 $+\mathrm{T}$ regulatory cells in the tumor microenvironment of human colorectal adenomas. Pathol Res Pract (2016) 212(2):106-12. doi: 10.1016/ j.prp.2015.12.002

93. Cui G. Immune battle at the premalignant stage of colorectal cancer: focus on immune cell compositions, functions and cytokine products. Am J Cancer Res (2020) 10(5):1308-20.

94. Wirta EV, Seppala T, Friman M, Vayrynen J, Ahtiainen M, Kautiainen H, et al. Immunoscore in mismatch repair-proficient and -deficient colon cancer. J Pathol Clin Res (2017) 3(3):203-13. doi: 10.1002/cjp2.71

95. Zhang P, Liu M, Cui Y, Zheng P, Liu Y. Microsatellite instability status differentially associates with intratumoral immune microenvironment in human cancers. Brief Bioinform (2020). doi: 10.1093/bib/bbaa180

96. Michel S, Benner A, Tariverdian M, Wentzensen N, Hoefler P, Pommerencke T, et al. High density of FOXP3-positive T cells infiltrating colorectal cancers with microsatellite instability. Br J Cancer (2008) 99 (11):1867-73. doi: 10.1038/sj.bjc.6604756

97. Giannakis M, Mu XJ, Shukla SA, Qian ZR, Cohen O, Nishihara R, et al. Genomic Correlates of Immune-Cell Infiltrates in Colorectal Carcinoma. Cell Rep (2016) 15(4):857-65. doi: 10.1016/j.celrep.2016.03.075

98. Tosolini M, Kirilovsky A, Mlecnik B, Fredriksen T, Mauger S, Bindea G, et al. Clinical impact of different classes of infiltrating $\mathrm{T}$ cytotoxic and helper cells (Th1, th2, treg, th17) in patients with colorectal cancer. Cancer Res (2011) 71 (4):1263-71. doi: 10.1158/0008-5472.CAN-10-2907

99. Le Gouvello S, Bastuji-Garin S, Aloulou N, Mansour H, Chaumette MT, Berrehar F, et al. High prevalence of Foxp3 and IL17 in MMR-proficient colorectal carcinomas. Gut (2008) 57(6):772-9. doi: 10.1136/gut.2007.123794

100. Langhans B, Nischalke HD, Kramer B, Dold L, Lutz P, Mohr R, et al. Role of regulatory $\mathrm{T}$ cells and checkpoint inhibition in hepatocellular carcinoma. Cancer Immunol Immunother (2019) 68(12):2055-66. doi: 10.1007/s00262019-02427-4

101. Bauer K, Nelius N, Reuschenbach M, Koch M, Weitz J, Steinert G, et al. T cell responses against microsatellite instability-induced frameshift peptides and influence of regulatory $\mathrm{T}$ cells in colorectal cancer. Cancer Immunol Immunother (2013) 62(1):27-37. doi: 10.1007/s00262-012-1303-8

102. Dyck L, Wilk MM, Raverdeau M, Misiak A, Boon L, Mills KH. Anti-PD-1 inhibits Foxp3(+) Treg cell conversion and unleashes intratumoural effector
$\mathrm{T}$ cells thereby enhancing the efficacy of a cancer vaccine in a mouse model. Cancer Immunol Immunother (2016) 65(12):1491-8. doi: 10.1007/s00262016-1906-6

103. Zhang H, Li Y, Liu X, Liang Z, Yan M, Liu Q, et al. ImmTAC/Anti-PD-1 antibody combination to enhance killing of cancer cells by reversing regulatory T-cell-mediated immunosuppression. Immunology (2018) 155 (2):238-50. doi: 10.1111/imm.12954

104. Yoshida K, Okamoto M, Sasaki J, Kuroda C, Ishida H, Ueda K, et al. AntiPD-1 antibody decreases tumour-infiltrating regulatory T cells. BMC Cancer (2020) 20(1):25. doi: 10.1186/s12885-019-6499-y

105. Wang Y, Wei B, Gao J, Cai X, Xu L, Zhong H, et al. Combination of Fruquintinib and Anti-PD-1 for the Treatment of Colorectal Cancer. J Immunol (2020) 205:2905-15. doi: 10.4049/jimmunol.2000463

106. Volpin V, Michels T, Sorrentino A, Menevse AN, Knoll G, Ditz M, et al. CAMK1D Triggers Immune Resistance of Human Tumor Cells Refractory to Anti-PD-L1 Treatment. Cancer Immunol Res (2020) 8(9):1163-79. doi: 10.1158/2326-6066.CIR-19-0608

107. Li N, Kang Y, Wang L, Huff S, Tang R, Hui H, et al. ALKBH5 regulates anti-PD-1 therapy response by modulating lactate and suppressive immune cell accumulation in tumor microenvironment Proc Natl Acad Sci U States America (2020) 117(33):20159-70. doi: 10.1073/pnas.1918986117

108. Nusrat M. Response to anti-PD-1 in microsatellite stable colorectal cancer: a STAT need. Clin Cancer Res (2020) 26:5775- 7. doi: 10.1158/10780432.CCR-20-2901

109. Shi H, Lan J, Yang J. Mechanisms of Resistance to Checkpoint Blockade Therapy. Adv Exp Med Biol (2020) 1248:83-117. doi: 10.1007/978-981-153266-5_5

110. Andrews A. Treating with Checkpoint Inhibitors-Figure \$1 Million per Patient. Am Health Drug Benefits (2015) 8(Spec Issue):9.

111. Guan Y, Kraus SG, Quaney MJ, Daniels MA, Mitchem JB, Teixeiro E. FOLFOX Chemotherapy Ameliorates CD8 T Lymphocyte Exhaustion and Enhances Checkpoint Blockade Efficacy in Colorectal Cancer. Front Oncol (2020) 10:586. doi: 10.3389/fonc.2020.00586

112. Schoonderwoerd MJA, Koops MFM, Angela RA, Koolmoes B, Toitou M, Paauwe M, et al. Targeting Endoglin-Expressing Regulatory T Cells in the Tumor Microenvironment Enhances the Effect of PD1 Checkpoint Inhibitor Immunotherapy. Clin Cancer Res (2020) 26(14):3831-42. doi: 10.1158/10780432.CCR-19-2889

113. Yuan W, Deng D, Jiang H, Tu C, Shang X, He H, et al. Hyperresponsiveness to interferon gamma exposure as a response mechanism to anti-PD-1 therapy in microsatellite instability colorectal cancer. Cancer Immunol Immunother (2019) 68(2):257-68. doi: 10.1007/s00262-018-2270-5

114. Cai X, Wei B, Li L, Chen X, Liu W, Cui J, et al. Apatinib enhanced anti-PD-1 therapy for colon cancer in mice via promoting PD-L1 expression. Int Immunopharmacol (2020) 88:106858. doi: 10.1016/j.intimp.2020.106858

115. Knudson KM, Hicks KC, Alter S, Schlom J, Gameiro SR. Mechanisms involved in IL-15 superagonist enhancement of anti-PD-L1 therapy. J Immunother Cancer (2019) 7(1):82. doi: 10.1186/s40425-019-0551-y

116. Hutmacher C, Gonzalo Nunez N, Liuzzi AR, Becher B, Neri D. Targeted Delivery of IL2 to the Tumor Stroma Potentiates the Action of Immune Checkpoint Inhibitors by Preferential Activation of NK and CD8(+) T Cells. Cancer Immunol Res (2019) 7(4):572-83. doi: 10.1158/2326-6066.CIR-180566

117. Emambux S, Tachon G, Junca A, Tougeron D. Results and challenges of immune checkpoint inhibitors in colorectal cancer. Expert Opin Biol Ther (2018) 18(5):561-73. doi: 10.1080/14712598.2018.1445222

Conflict of Interest: The author declares that the research was conducted in the absence of any commercial or financial relationships that could be construed as a potential conflict of interest.

Copyright (C) 2021 Cui. This is an open-access article distributed under the terms of the Creative Commons Attribution License (CC BY). The use, distribution or reproduction in other forums is permitted, provided the original author(s) and the copyright owner(s) are credited and that the original publication in this journal is cited, in accordance with accepted academic practice. No use, distribution or reproduction is permitted which does not comply with these terms. 